\title{
LEGENDRE-TYPE RELATIONS FOR GENERALIZED COMPLETE ELLIPTIC INTEGRALS
}

\author{
SHINGO TAKEUCHI
}

Abstract. Legendre's relation for the complete elliptic integrals of the first and second kinds is generalized. The proof depends on an application of the generalized trigonometric functions and is alternative to the proof for Elliott's identity.

Mathematics subject classification (2010): 33E05, 33C75, 34L10.

Keywords and phrases: Legendre's relation, complete elliptic integrals, generalized trigonometric functions, Elliott's identity.

\section{REFERENCES}

[1] G. D. Anderson, S. L. Qiu, M. K. Vamanamurthy and M. Vuorinen, Generalized elliptic integrals and modular equations, Pacific J. Math. 192, 1 (2000), 1-37.

[2] G. Andrews, R. Askey And R. Roy, Special functions, Encyclopedia of Mathematics and its Applications, 71. Cambridge University Press, Cambridge, 1999.

[3] B. A. BHAYO AND L. YIN, On generalized $(p, q)$-elliptic integrals, preprint, arXiv:1507.00031.

[4] J. M. Borwein And P. B. BorWein, Pi and the AGM, A study in analytic number theory and computational complexity. Reprint of the 1987 original. Canadian Mathematical Society Series of Monographs and Advanced Texts, 4. A Wiley-Interscience Publication. John Wiley \& Sons, Inc., New York, 1998

[5] P. DRÁbEK AND R. MANÁsEVICH, On the closed solution to some nonhomogeneous eigenvalue problems with $p$-Laplacian, Differential Integral Equations 12 (1999), 773-788.

[6] P. DurEn, The Legendre relation for elliptic integrals, Paul Halmos, 305-315, Springer, New York, 1991.

[7] E. B. Elliott, A formula including Legendre's $E K^{\prime}+K E^{\prime}-K K^{\prime}=\frac{1}{2} \pi$, Messenger Math. 33 (1903/1904), 31-32.

[8] A. Erdélyi, W. Magnus, F. Oberhettinger and F. G.Tricomi, Higher transcendental functions. Vol. I, Based on notes left by Harry Bateman. With a preface by Mina Rees. With a foreword by E. C. Watson. Reprint of the 1953 original. Robert E. Krieger Publishing Co., Inc., Melbourne, Fla., 1981.

[9] T. Kamiya And S. TAKeUCHI, Complete $(p, q)$-elliptic integrals with application to a family of means, preprint, arXiv:1507.01383.

[10] J. LANG AND D. E. EDMUNDS, Eigenvalues, embeddings and generalised trigonometric functions, Lecture Notes in Mathematics, 2016. Springer, Heidelberg, 2011.

[11] F. W. J. Olver, D. W. Lozier, R. F. Boisvert, AND C. W. Clark (Editors), NIST Handbook of Mathematical Functions, [With 1 CD-ROM (Windows, Macintosh and UNIX)], US Department of Commerce, National Institute of Standards and Technology, Washington, D.C., 2010; Cambridge University Press, Cambridge, London and New York, 2010.

[12] S. TAKEUCHI, Generalized Jacobian elliptic functions and their application to bifurcation problems associated with p-Laplacian, J. Math. Anal. Appl. 385, 1 (2012), 24-35.

[13] S. TAKeuchi, The basis property of generalized Jacobian elliptic functions, Commun. Pure Appl. Anal. 13, 6 (2014), 2675-2692.

[14] S. TAKEUCHI, A new form of the generalized complete elliptic integrals, Kodai Math. J. 39, 1 (2016), $202-226$. 
[15] S. TAKeUCHI, Complete p-elliptic integrals and a computation formula of $\pi_{p}$ for $p=4$, preprint, arXiv:1503.02394.

[16] L. YIN AND L.-G. HUANG, Inequalities for the generalized trigonometric and hyperbolic functions with two parameters, J. Nonlinear Sci. Appl. 8, 4 (2015), 315-323. 